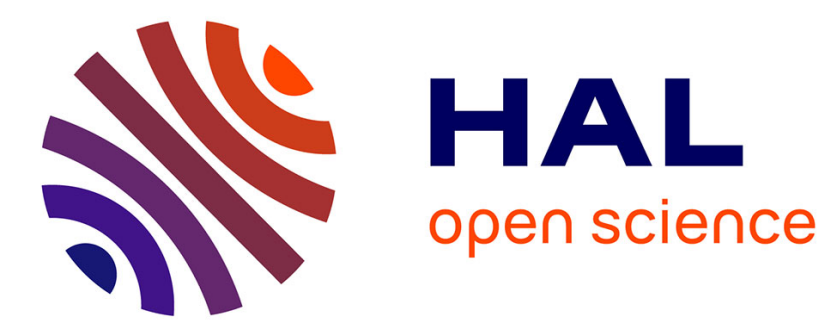

\title{
Three-dimensional characterization of strain localization bands in high-resolution elastoplastic polycrystals
}

\author{
Fabrice Barbe, Romain Quey, Andrey Musienko, Georges Cailletaud
}

\section{To cite this version:}

Fabrice Barbe, Romain Quey, Andrey Musienko, Georges Cailletaud. Three-dimensional characterization of strain localization bands in high-resolution elastoplastic polycrystals. Mechanics Research Communications, 2009, 36, pp.762-768. 10.1016/j.mechrescom.2009.06.002 . hal-00423757

\section{HAL Id: hal-00423757}

https://hal-mines-paristech.archives-ouvertes.fr/hal-00423757

Submitted on 12 Feb 2021

HAL is a multi-disciplinary open access archive for the deposit and dissemination of scientific research documents, whether they are published or not. The documents may come from teaching and research institutions in France or abroad, or from public or private research centers.
L'archive ouverte pluridisciplinaire HAL, est destinée au dépôt et à la diffusion de documents scientifiques de niveau recherche, publiés ou non, émanant des établissements d'enseignement et de recherche français ou étrangers, des laboratoires publics ou privés. 


\title{
Three-dimensional characterization of strain localization bands in high resolution elastoplastic polycrystals
}

\author{
Fabrice Barbe $^{1,2, *}$, Romain Quey ${ }^{3}$, Andrei Musienko ${ }^{4}$, Georges Cailletaud $^{2}$ \\ (1) INSA Rouen, Groupe de Physique des Matériaux, CNRS UMR 6634 \\ 76801 Saint Etienne du Rouvray, France \\ (2) Mines Paristech, Centre des Matériaux, CNRS UMR 7633 \\ 91003 Evry, France \\ (3) Ecole des Mines de Saint Etienne, Laboratoire PECM, CNRS UMR 5146 \\ 42023 Saint Etienne, France \\ (4) Material Strength Department, State Polytechnical University, 195251 \\ St-Petersburg, Russia
fabrice.barbe@insa-rouen.fr
quey@emse.fr
georges.cailletaud@ensmp.fr \\ andrei.musienko@mat.ensmp.fr
}

(*) Corresponding author: Fabrice BARBE

INSA Rouen, GPM UMR 6634, BP08, Avenue de l'Université

76801 Saint Etienne du Rouvray Cedex, France

tel: 332329597 60, fax: 33232959704

\begin{abstract}
In crystalline materials, the experimental observation of the localization of plastic strains in particular directions is generally restricted to the surface of a sample containing some hundreds of grains, because of the difficulties underlying microstructural analysis. In these conditions, the determination of the morphological characteristics of localization can be limited by the poor statistical representativity of the domain of observation. The purpose of this work is to extend the analysis of localization -localization bands or elseto the three-dimensional elastoplastic strain fields of a high resolution representative volume element of a polycrystal.
\end{abstract}

Keywords: Strain localization, Crystal plasticity, 3D polycrystal, Finite elements

\section{Introduction}

Elastoplastic strains in polycrystals are known to localize in the form of bands which are oriented in the direction of maximum shear stress, i.e. at $45^{\circ}$ with respect to the uniaxial tension direction. Such bands can be observed at the surface of plastically deformed polycrystals, either experimentally (Doumalin et al., 2000; Doumalin et al., 2003; Gélébart et al., 2004; Héripré et al., 2007; St-Pierre et al., 2008) or by numerical simulations (Cordier et al., 2005; Zeghadi et al., 2007; Osipov et al., 2008; Lebensohn et al., 2009). They appear at the early stages of plasticity and can expand over few grains with a width of the order of the grain size or less. Some features of their morphology (orientation and length) have been determined in 2D by means of point-to-point covariance analysis of strain fields measured by SEM microextensometry (Doumalin, 2000; Doumalin et al., 2000; Doumalin et al., 2003): once segmented into two classes corresponding to lower and higher strains, the strain field is considered as a 
two-phase medium whose morphology is analyzed in terms of covariance. The point-topoint covariance provides the probability to find a given phase at two points separated by a given vector; it thus enables to detect any periodic repetition of patterns in the spatial distribution of field variables and more generally to determine the range of the interactions between grains in a polycrystal.

Such information can be a prevailing factor for the relevance of modellings based on microstructural morphology: the size of the representative volume element, the depth over which boundary conditions affect the local behavior, the distance over which fields become uncorrelated, ... and the degree of accuracy of the morphological features which should be taken into account might depend on it. As current experimental techniques cannot, to this day, give access to this kind of information concerning a large threedimensional polycrystal, it is proposed to extract this information from FE simulations of high resolution 3D polycrystals, that provide full-field solutions for the elastoplastic intracrystalline stresses and strains in a large number of grains. This numerical approach has been proved to be successfull in reproducing the intracrystalline strain localization experimentally measured on the surface of a multicrystal, provided that the mesh is fine enough (Musienko et al., 2007; Héripré et al., 2007; Zhao et al., 2008). For the present study of the localization in a 3D polycrystalline medium mimicking a real microstructure with its random morphological nature, we resort to Voronoi tessellations of space and a fine discretization of each Voronoi polyhedron into several tens of elements, so that local fields inside each grain be accessible (Barbe et al., 2001a; Barbe et al., 2001b).

Section 2 recalls the principles of the numerical modelling and presents contour plots of axial stress and strain. The process of computing the point-to-point covariance of a segmented variable is described briefly in section 3 . Then $2 \mathrm{D}$ and $3 \mathrm{D}$ covariance fields are analyzed in section 4 .

\section{Numerical simulation}

Data for the analysis of stress and strain intracrystalline localization in elastoplastic polycrystals are obtained from FE simulations. The behavior of each integration point of the FE mesh is described by a constitutive model which was first introduced in (Méric et al., 1991). It has then been extensively used in several works which have laid an important groundwork (Barbe et al., 2001a; Diard et al., 2005; Sai et al., 2006; Osipov et al., 2008) and more particularly for the direct comparison of experimental and simulated intracrystalline strain fields in (Musienko et al., 2007).

The constitutive model is based on the Schmid law: the plastic strain ${\underset{\sim}{g}}^{g}$ results from the slip on the crystallographic slip systems $\gamma^{\mathrm{s}}$ (eq. 1); the onset of slip and the slip rate are determined in eq. 2 from the resolved shear $\operatorname{stress} \tau^{\mathrm{s}}$ and the hardening variables $r^{\mathrm{s}}$ and $x^{\mathrm{s}}$ (isotropic and kinematic hardenings, respectively). Whereas $\tau^{\mathrm{s}}$ directly results from the state of stress $\underset{\sim}{\sigma}$ and the Schmid tensor $\underset{\sim}{m^{\mathrm{s}}}$ (eq. 3, where $\underline{n}^{\mathrm{s}}$ is the unit vector normal to the slip plane and $\underline{l}^{\mathrm{s}}$ is the slip direction), the hardening variables follow non-linear evolutions defined by classical phenomenological formulations of hardening (eq. 4,5). The material parameters are those of a Ni-based alloy which has been under consideration in (Barbe et al., 2001a); it has an FCC structure (slip on the $\{111\}\langle 110\rangle$ octahedral slip systems). 


$$
\begin{gathered}
\dot{\gamma}^{\mathrm{s}}=\dot{v}^{\mathrm{s}} \operatorname{sign}\left(\tau^{\mathrm{s}}-x^{\mathrm{s}}\right) \quad ; \quad{\underset{\sim}{\dot{\varepsilon}}}^{\mathrm{g}}=\sum_{s}{\underset{\sim}{m}}_{m^{\mathrm{s}}} \dot{\gamma}^{\mathrm{s}} \\
\dot{v}^{\mathrm{s}}=\left\langle\frac{\left|\tau^{\mathrm{s}}-x^{\mathrm{s}}\right|-r^{\mathrm{s}}}{K}\right\rangle^{n} \quad \text { with }\langle x\rangle=\operatorname{Max}(x, 0) \quad \text { and } v^{\mathrm{s}}\left(t=t_{0}\right)=0 \\
\tau^{\mathrm{s}}=\underset{\sim}{\sigma}:{\underset{\sim}{m}}^{\mathrm{s}}=\frac{1}{2} \underset{\sim}{\sigma}:\left(\underline{n}^{\mathrm{s}} \otimes \underline{l}^{\mathrm{s}}+\underline{l}^{\mathrm{s}} \otimes \underline{n}^{\mathrm{s}}\right) \\
x^{\mathrm{s}}=c \alpha^{\mathrm{s}} ; r^{\mathrm{s}}=R_{0}+Q \sum_{r} h_{r s}\left\{1-e^{-b v^{\mathrm{r}}}\right\} \\
\dot{\alpha}^{\mathrm{s}}=\dot{\gamma}^{\mathrm{s}}-d \alpha^{\mathrm{s}} \dot{v}^{\mathrm{s}} \text { with } \alpha^{\mathrm{s}}\left(t=t_{0}\right)=0
\end{gathered}
$$

At the current state of the study, the polycrystalline domains of simulation, with cubic shape, contain $13 \times 13 \times 13=2197$ grains. According to previous analyses in (Barbe et al., 2001b), any integration point at a distance lower than 2 Mean Grain Size (MGS) from the polycrystal boundaries must be excluded from the analysis to avoid any boundary effect. The remaining region at the centre is large enough $(9 \times 9 \times 9=729$ grains $)$ to provide representative properties of the polycrystalline material at the mean polycrystal scale, as checked in the previously cited studies based on the same modelling. As a major improvement regarding these previous studies, the polycrystals microstructural morphologies are obtained from Voronoi tessellations which are slightly modified prior to free meshing. This procedure, which enables a rapid and robust meshing without local overrefinements, is implemented in the NePeR software (NePeR, 2007; Quey and Barbe, 2007). The resulting meshes conform to polyhedra boundaries, as illustrated on fig.1a. The polycrystals in this study, made of 2197 grains, are discretized into 180000 tetrahedral quadratic elements, which leads to an average of 82 elements per grain. This mesh density respects the conditions under which local fields are unaffected by the type and number of elements (Barbe et al., 2001c; Osipov et al., 2008). FE simulations are performed with the software Zset (Mines Paristech, ONERA, nwnumerics.com, (Besson et al., 1998)) on a Linux cluster, using an implicit method for the integration of the constitutive laws and a Newton type method for the resolution of the non-linear problem.

The polycrystals are subjected to uniaxial tension up to a total imposed axial strain of the order $1 \%$. Their lateral boundaries are left free of mean stress; they are also imposed to remain parallel to their initial positions, such as to induce a boundary effect similar, in average, to the one which would be exerted by surrounding grains of the medium from which the considered polycrystal is extracted. The obtained axial stress and axial strain contour plots are presented on fig.1b,c. The main following classical features can be noticed: both stress and strain are heterogeneous at the scale of grains as well as inside grains; strain localization regions do not systematically correspond to stress localization regions and their patterns do not seem to be the same; strain seems to localize in the form of bands oriented at $45^{\circ}$ with respect to the tension direction (vertical, on the figure).

\section{Statistical treatments}

Given a field variable, say axial strain $\varepsilon(\underline{X})$ where $\underline{X}=\left(x_{1}, x_{2}, x_{3}\right)$ is the position vector in the polycrystal frame, and an associated threshold $\varepsilon_{t}$, the strain field is first segmented 

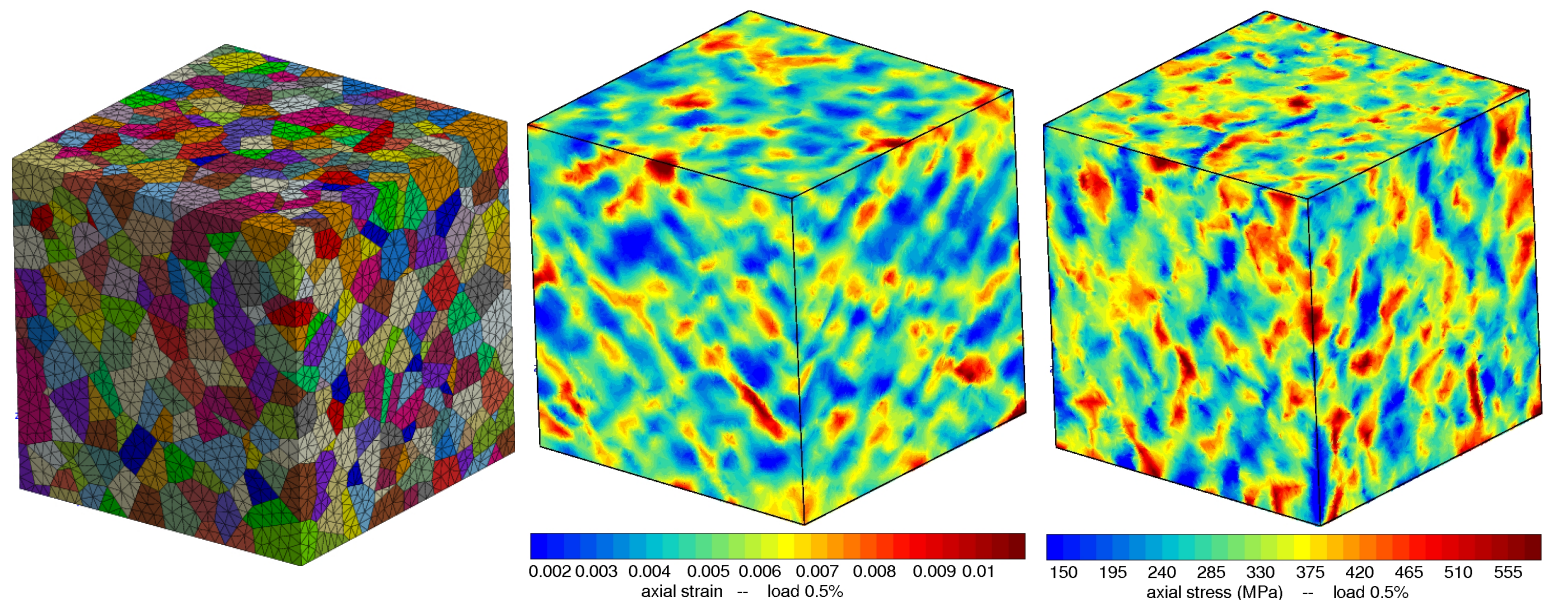

Figure 1: Strain and stress fields in a high resolution polycrystal submitted to $0.5 \%$ uniaxial tension. (Left: a) Polycrystal morphology and mesh (2197 grains freely meshed into 180000 tetrahedral elements). (Middle: b) axial strain; (Right: c) axial stress. Colors on the online version.

into two sets of points: those where $\varepsilon<\varepsilon_{t}$ and the others, forming point clouds that will be denoted phase $\mathcal{B}=\left\{\underline{X} / \varepsilon(\underline{X}) \geq \varepsilon_{t}\right\}$. The $3 \mathrm{D}$ visualization of these points does not provide particular information on the localization of higher strains: they form more or less large connex regions in the polycrystal space which seem to be equiprobably present and to propagate in different directions. The axial stress shows the same features. So, the characterization of stress and strain localization based on direct visualization of segmented variables can only be qualitative.

We thus resort to a morphological analysis, the point-to-point covariance, which enables the characterization of two-phase materials or any binary medium. It is defined according to a vector $\underline{h}$ and corresponds to the probability that a point and its translated according to $\underline{h}$ belong to the same phase. Let $f_{\mathcal{B}}(\underline{X})$ be the characteristic function of the phase $\mathcal{B}$.

$$
f_{\mathcal{B}}(\underline{X})=\left\{\begin{array}{l}
1 \text { if } \underline{X} \in \mathcal{B} \\
0 \text { else }
\end{array}\right.
$$

Following the formulation of point-to-point covariance in a continuous space, in a discrete space as the one provided by the set of integration points of the polycrystal, the covariance $S_{\varepsilon_{t}}(\underline{h})$ of $\mathcal{B}$ is written as a function of the translation vector $\underline{h}$ :

$$
S_{\varepsilon_{t}}(\underline{h})=\frac{1}{\prod_{i=1}^{3}\left(L-h_{i}\right)} \sum_{i=1}^{3} \sum_{x_{i}=0}^{L-h_{i}} f_{\mathcal{B}}(\underline{X}) f_{\mathcal{B}}(\underline{X}+\underline{h})
$$

where $\underline{h}=\left(h_{i}\right)_{i=1,3}, L$ is the length of the edge of the cubic aggregate. The sum $\sum_{i=1}^{3} \sum_{x_{i}=0}^{L-h_{i}}$ corresponds to the sum over all the points within the range $\left[0, L-h_{1}\right] \times$ $\left[0, L-h_{2}\right] \times\left[0, L-h_{3}\right]$ i.e. to the volume average over the intersection set $\{\underline{X}\} \cap\{\underline{X}+\underline{h}\}$. 


\section{Analysis}

The obtained covariance field exhibits a central symmetry over $\underline{h}=\underline{0}$ since a translation according to $\underline{h}$ or $-\underline{h}$ leads to the same intersection set. Taking MGS as the length scale, the covariance is defined in the range $[-13,13] \times[-13,13] \times[-13,13]$ since the polycrystals under concern in this work contains $13 \times 13 \times 13$ grains. In order to make a qualitative comparison with the experimental characterizations of (Doumalin, 2000; Doumalin et al., 2003) on a two-phase material and on a zirconium alloy under tension, the covariance field of axial strain is first visualized in two perpendicular planes of the translation space $\left(h_{1}, h_{2}, h_{3}\right)$ which include the tensile axis $\left(x_{3}\right):\left(h_{1}=0\right)$ in fig. $2 \mathrm{a}$ and $\left(h_{2}=0\right)$ in fig. $2 \mathrm{~b}$. For both plots, high values of covariance appear close to the centre of the translation space: at $\underline{h}=\underline{0}$, the probability of finding the same phase $\mathcal{B}$ is maximum and $S_{\varepsilon_{t}}(\underline{0})$ takes the value of the volume fraction of the phase $\mathcal{B}$ (here, 0.5). For small distances $h, S_{\varepsilon_{t}}$ remains high with a spherical symmetry; this short distance isotropic localization is the consequence of local interactions independent of the microstructure, as in any FE simulation with fine enough mesh. With $h$ from 1 up to 3 , the highest values of $S_{\varepsilon_{t}}$ are distributed according to a cross with branches oriented at $45^{\circ}$ with respect to the tension direction (vertical) and the smallest values appear at $0^{\circ}$ and $90^{\circ}$ angles to the tension direction. Similar characterizations can be made concerning equivalent strain or the accumulated plastic strain. This indicates that localization of highest strains will more probably happen in the form of bands, 2 or 3 MGS long and oriented at $45^{\circ}$ to the tension axis, which is consistent with experimental observations of Doumalin et al on a two-phase material and a zirconium polycrystal. Furthermore, axial strain is unlikely to localize in bands parallel or perpendicular to the tensile direction. For $h>3$, the distributions of $S_{\varepsilon_{t}}$ differ from one plane to the other, which means that the isotropy of the problem, though it is accurately respected at the mean polycrystal scale, reaches its limits at the local scale, as soon as the analysis of the interactions between grains is performed above 4 MGS. This limit can evidently be extended with larger polycrystals, but it is not in the scope of this paper. As $h$ is increased, the size of the intersection set of points decreases, thus directly affecting the statistical representativity for the estimation of the covariance. For this reason and as however local fields are influenced by the boundary conditions on a depth of the order of 2 or 3 MGS, any analysis for $h \geq 10$ should be avoided with this size of polycrystal.

In fig 3a, a 3D visualization of the covariance field is performed by means of isointensity surfaces corresponding to values of covariance above the one that distinctly delimits the cross in the 2D visualizations, taken at 0.24 . Decreasing the value of $S_{\varepsilon_{t}}$ from its maximum 0.5 , the shapes of the isointensity surfaces vary from a sphere to kind of a hourglass and then takes the shape of a three-dimensional cross whose eight branches are the diagonals of a parallelepiped with a length of the order of 3 MGS, thus no longer respecting an axial symmetry. As nothing at this state enables to ensure the statistical representativity of the strain patterns for $h \geq 3$, the interpretation of the geometry of this eight-branches cross would require great care. The hourglass-like shape of isointensity surfaces is however confirmed in fig 3b, where the covariance field is obtained by ensemble averaging over three covariance fields obtained from three different 2197-grains polycrystals. This shape is characteristic of an equiprobable localization of the highest strains in all directions at a $45^{\circ}$ angle from the tension direction. 

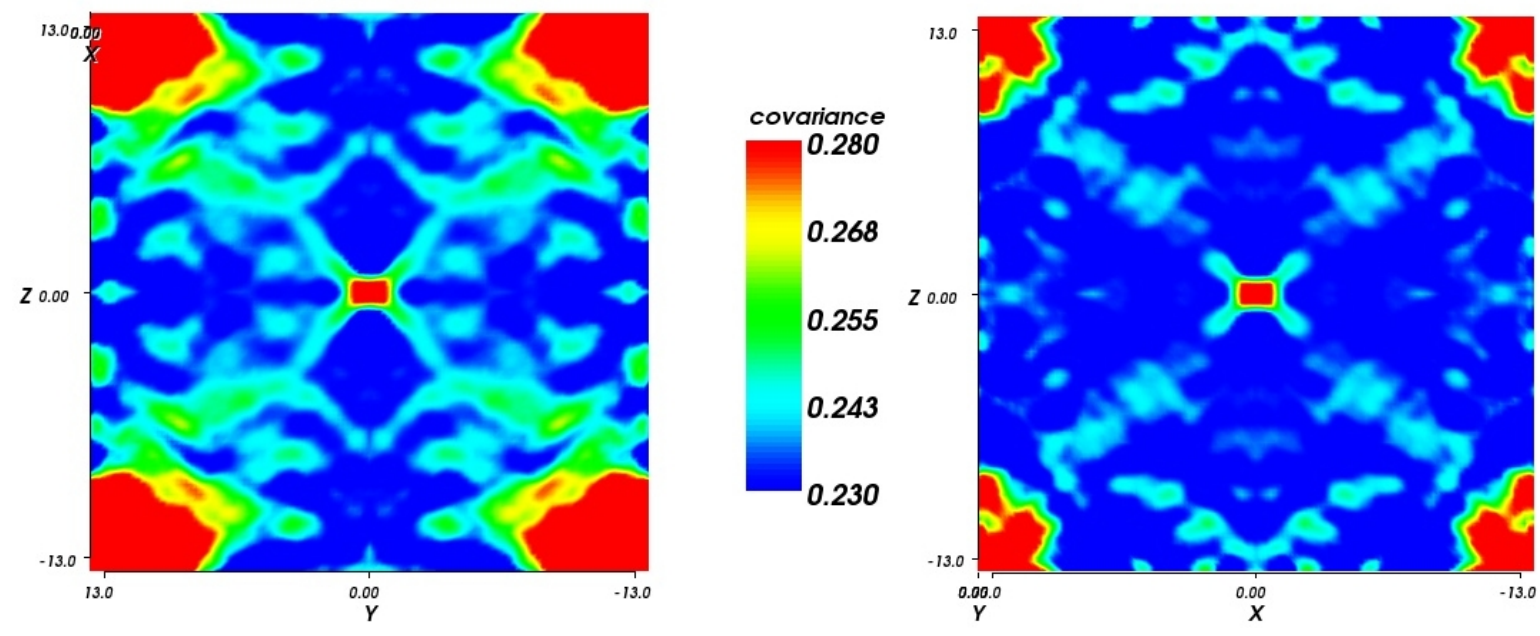

Figure 2: Covariance of segmented axial strain for translations in all the directions of two planes containing the tensile axis $\left(x_{3}\right)$ : $\left(h_{1}=0\right)$ (Left: a) and $\left(h_{2}=0\right)$ (Right: b). The length scale of the axis are given in terms of mean grain size. Colors on the online version.
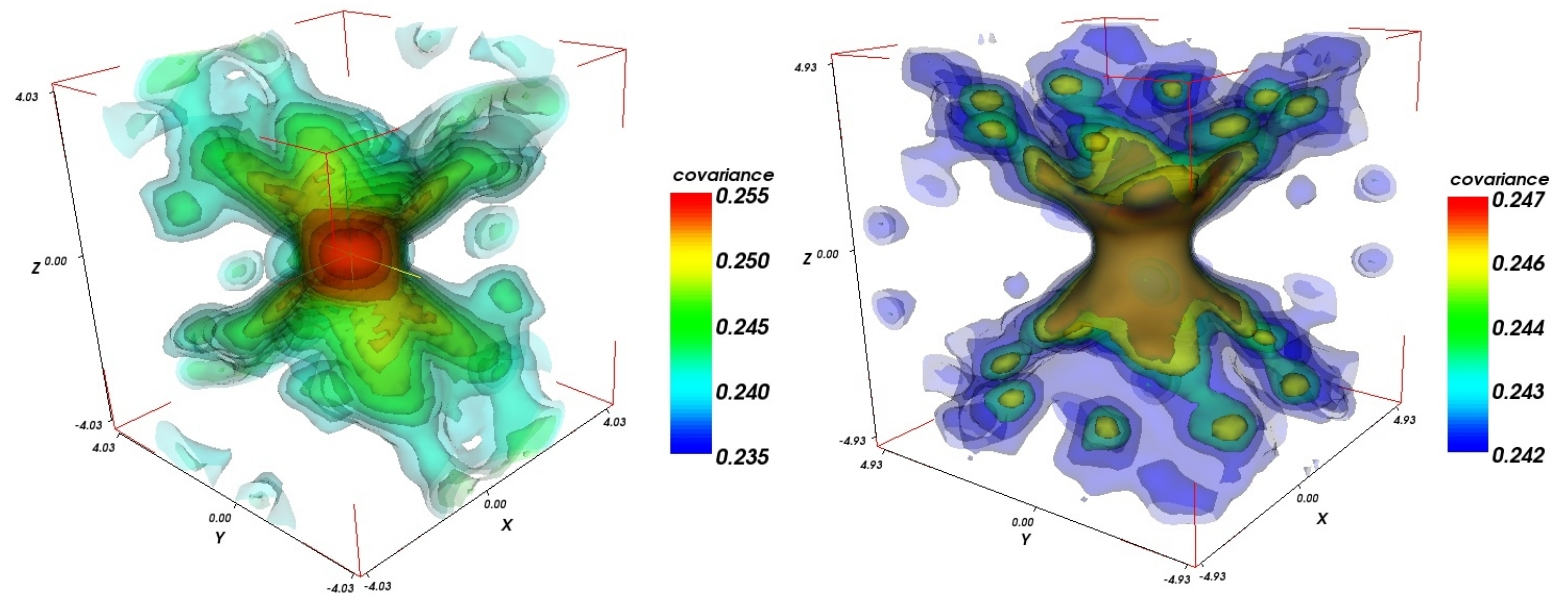

Figure 3: Isointensity surfaces of covariance of segmented axial strain in the threedimensional translation space: from a single 2197-grains polycrystal (Left: a) and obtained by ensemble averaging over three 2197-grains polycrystals (Right: b). In both cases selected, covariances are superior to 0.24 . Colors on the online version. 


\section{Conclusion}

The characterization of the three-dimensional distribution of highest strains in an elastoplastic polycrystal has been performed by means of point-to-point covariance of segmented strain field which was computed in a polycrystal with high intracrystalline resolution. It has shown the propensity for strains to localize equiprobably along bands 2 or 3 MGS long and oriented at around $45^{\circ}$ with respect to the tensile direction. As to this day experimental investigations can hardly provide measures which are statistically representative of three-dimensional localization, this work constitutes the first step ever towards any such detailed and systematic 3D characterization. Future work will extend the analysis to different loadings and materials computed on larger polycrystals and, besides, should refine the characterization as in (Doumalin et al., 2003) by resorting to a correlation function based on real values of strains instead of segmented values.

\section{Acknowledgements}

Thanks are due to Guido Lodato (CORIA, CNRS INSA Rouen) for his selfless introduction to the Paraview visualization application. CRIHAN (Centre de Ressources Informatiques de HAute Normandie) is acknowledged for providing computing ressources (Project $N^{\circ} 2008012$ ).

\section{References}

Barbe, F., Decker, L., Jeulin, D., and Cailletaud, G. (2001a). Intergranular and intragranular behavior of polycrystalline aggregates. Part 1: F.E. model. Int $J$ Plasticity, 17:513-536.

Barbe, F., Forest, S., and Cailletaud, G. (2001b). Intergranular and intragranular behavior of polycrystalline aggregates. Part 2: results. Int J Plasticity, 17:537563.

Barbe, F., Forest, S., and Cailletaud, G. (2001c). Polycrystalline plasticity under small strains. Toward finer descriptions of microstructures. In E. Bouchaud et al, editor, NATO Proceedings, Physical Aspects of Fracture, pages 191-206. Kluwer Academic Publishers.

Besson, J., Leriche, R., Foerch, R., and Cailletaud, G. (1998). Object-Oriented Programming Applied to the Finite Element Method. Part II. Application to Material Behaviors. Revue Européenne des Éléments Finis, 7(5):567-588.

Cordier, P., Barbe, F., Durinck, J., Tommasi, A., and Walker, A. (2005). Plastic Deformation of mantle minerals: Multiscale numerical modelling, volume 7 , chapter 16, pages 389-415. 7th EMU School of Mineralogy: Mineral behaviour at extreme conditions, Heidelberg, Germany, June 2005.

Diard, O., Leclercq, S., Rousselier, G., and Cailletaud, G. (2005). Evaluation of finite element based analysis of 3D multicrystalline aggregates plasticity. Application to crystal plasticity model identification and the study of stress and strain fields near grain boundaries. Int J Plasticity, 21:691-722.

Doumalin, P. (2000). Microextensométrie locale par corrélation d'images numériques. $\mathrm{PhD}$ thesis, Ecole Polytechnique. 
Doumalin, P., Bornert, M., and Crépin, J. (2003). Characterisation of the strain distribution in heterogeneous materials. Mécanique \& Industries, 4:607-617.

Doumalin, P., Bornert, M., and Soppa, E. (2000). Computational and experimental investigations of the local strain field in elastoplastic two-phase materials. In Advances in mechanical behaviour, plasticity and damage, Proc. Euromat 2000, pages 323-328. Elsevier.

Gélébart, L., Crépin, J., Dexet, M., Sauzay, M., and Roos, A. (2004). Identification of Crystalline Behavior on Macroscopic Response and Local Strain Field Analysis: Application to Alpha Zirconium Alloys. J ASTM Int, 1(9):JAI12333.

Héripré, E., Dexet, M., Crépin, J., Gélébart, L., Roos, A., Bornert, M., and Caldemaison, D. (2007). Coupling between experimental measurements and polycrystal finite element calculations for micromechanical study of metallic materials. Int $J$ Plasticity, 23:1512-1539.

Lebensohn, R., Montagnat, M., Mansuy, P., Duval, P., Meysonnier, J., and Philip, A. (2009). Modeling viscoplastic behavior and heterogeneous intracrystalline deformation of columnar ice polycrystals. Acta Mater, 57:1405-1415.

Méric, L., Poubanne, P., and Cailletaud, G. (1991). Single Crystal Modeling for Structural Calculations. Part 1: Model Presentation. J Engng Mat Technol, 113:162-170.

Musienko, A., Tatschl, A., Schmidegg, K., Pippan, R., and Cailletaud, G. (2007). Threedimensional finite element simulation of a polycrystalline copper specimen. Acta Mater, 55:4121-4136.

NePeR (2007). NePeR: a software to generate random polycrystals for the finite element method (version 1.6). http://www.emse.fr/ quey/neper.

Osipov, N., Gourgues-Lorenzon, A., Marini, B., Mounoury, V., Nguyen, F., and Cailletaud, G. (2008). FE modelling of bainitic steels using crystal plasticity. Phil Mag, 88(30):3757-3777.

Quey, R. and Barbe, F. (2007). Free meshing of microstructures based on modified Voronoi tessellations. In 17th Int Workshop Computational Mechanics of Materials, Paris (France), August 22-24.

Sai, K., Cailletaud, G., and Forest, S. (2006). Micro-mechanical modeling of the inelastic behavior of directionally solidified materials. Mech Materials, 38:203-217.

St-Pierre, L., Héripré, E., Dexet, M., Crépin, J., Bertolino, G., and Bilger, N. (2008). 3D simulations of microstructure and comparison with experimental microstructure coming from O.I.M. analysis. Int J Plasticity, 24:1516-1532.

Zeghadi, A., Forest, S., Gourgues, A., and Bouaziz, O. (2007). Ensemble averaging stressstrain fields in polycrystalline aggregates with a constrained surface microstructure - Part 2: crystal plasticity. Phil Mag, 87:1425-1446.

Zhao, Z., Ramesh, M., Raabe, D., Cuitiño, A., and Radovitzky, R. (2008). Investigation of three-dimensional aspects of grain-scale plastic surface deformation of an aluminium oligocrystal. Int J Plasticity, 24:2278-2297. 